\title{
DETECTION OF SINGLE SUBMILLIMETER-WAVE PHOTONS USING QUANTUM DOTS
}

\author{
S. Komiyama ${ }^{a}, \mathrm{O} \operatorname{Astafiev}^{b}$, V. Antonov ${ }^{b, *}$ And T. Kutsuwa ${ }^{a}$ \\ ${ }^{a}$ Department of Basic Science, University of Tokyo \\ Komaba 3-8-1, Meguro-ku, Tokyo, Japan \\ ${ }^{b}$ Japan Science and Technology Corporation (JST) \\ Kawaguchi, Saitama 332-0012, Japan
}

Single-photon detection in a range of submillimeter waves $(\lambda=$ $0.17-0.20 \mathrm{~mm}$ ) is demonstrated by using lateral semiconductor quantum dots fabricated on a high-mobility GaAs/AlGaAs single heterostructure crystal. When a submillimeter photon is absorbed by the quantum dot while it is operated as a single-electron transistor, it switches on (or off) the conductance through the quantum dot. An incident flux of 0.1 photons $/ \mathrm{s}$ on an effective detector area, $(0.1 \mathrm{~mm})^{2}$, is detected with a $1 \mathrm{~ms}$ time resolution. The effective noise equivalent power is roughly estimated to reach on the order of $10^{-22} \mathrm{~W} / \mathrm{Hz}^{1 / 2}$, a value superior to the ever reported best values of conventional detectors by a factor more than $10^{4}$.

PACS numbers: $72.20 . \mathrm{Ht}, 73.40 . \mathrm{Hm}, 76.40 .+\mathrm{b}$

\section{Introduction}

Direct detection of extremely weak submillimeter waves has been a nontrivial challenge for experimentalists due to limited sensitivities of available detectors. In terms of the noise equivalent power (NEP), best reported values of conventional detectors have been on the order of $10^{-18} \mathrm{~W} / \mathrm{Hz}^{1 / 2}[1-3]$, which implies that one needs an incident flux of $10^{4}$ photons/s for the detection with a signal-to-noise ratio of unity in $1 \mathrm{~s}$ integration time. The effective sensitivity further degrades seriously at high modulation speeds, say, at $1 \mathrm{kHz}$. Increasing demand for more sensitive and faster submillimeter-wave detectors in the broad range of science and technology has given impetus to improvement of conventional detectors [1-3] as well as to innovation of device concept [4]. Nevertheless, sensitivity on a level of single photon detection has not been achieved. Here, we demonstrate single-photon detection in the submillimeter-wave range [5-7]. In our detection scheme, an incident flux of $10^{-1}$ photons/s on an effective detector area, $(0.1 \mathrm{~mm})^{2}$, is detected with a $1 \mathrm{~ms}$ time resolution.

*Present address: Royal Holloway, University of London, Egham, Surrey TW20 OEX, U.K. 


\section{Background}

A single-electron transistor (SET) is a device consisting of a small conducting island that is weakly coupled to outside reservoirs through tunneling [8]. The conduction through the island is usually blocked because adding one electron to the island costs additional electrostatic energy (the charging energy). Finite conduction is possible only if the electrochemical potential of the island lines up with that of the reservoirs. This is called the conductance resonance, which yields a series of conductance peaks as the electrochemical potential of the island is scanned by controlling an appropriate external parameter, say, the gate voltage.

In our SET, a relatively large semiconductor quantum dot (QD) serves as the conducting island. As shown in the scanning electron microscopy (SEM) photograph of Fig. 1a, the sample is fabricated on a high-mobility GaAs/AlGaAs single heterostructure crystal $\left(\mu_{\mathrm{H}}=80 \mathrm{~m}^{2} /(\mathrm{V} \mathrm{s})\right.$ and $\left.n_{\mathrm{s}}=2.4 \times 10^{15} / \mathrm{m}^{2}\right)$. A QD is formed by negatively biasing all the metal gates and depleting the two-dimensional electron gas (2DEG) from the regions underneath the gates. The $\mathrm{QD}$ is weakly coupled to the larger 2DEG regions outside the QD through tunnel barriers formed between the upper gate and the lower-left and the lower-right gates. The lower gate at the middle is used to control the electrochemical potential of the $\mathrm{QD}$.

(a)

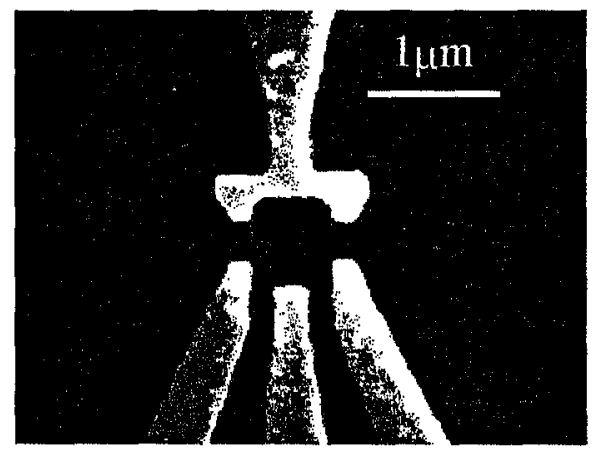

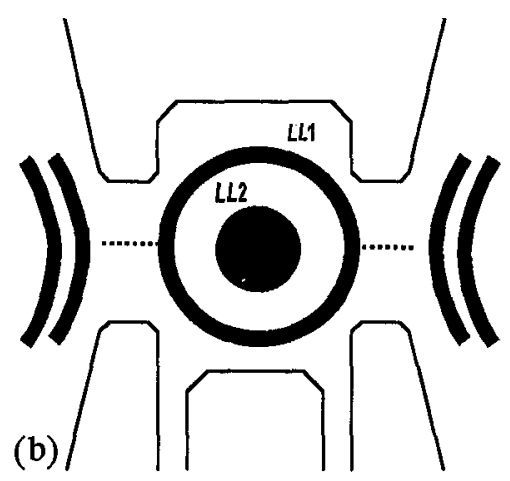

Fig. 1. (a) SEM micrograph of the QD. (b) Schematic view of the QD. The dark regions indicate metallic "inner-core" and "outer-ring" formed by the lowest two LLs.

To make the QD an efficient absorber of submillimeter waves and to achieve efficient photon-to-charge conversion, we apply strong magnetic fields $B$ normal to the plane of the QD. The resulting quantization of electron energy states into the Landau levels with energy spacing $\omega_{\mathrm{c}}$ makes the electron system a strong absorber of submillimeter waves with photon energy nearly equal to $\omega_{c}$. We specifically chose the strength of the magnetic field so that the lowest orbital Landau level, LL1 (with two opposite spin polarizations), is filled while the first excited Landau level, LL2, is lightly occupied with a small number of electrons as schematically shown in Fig. 1b and Fig. 2a. In this condition, the LL1 and the LL2 form two compressible metallic regions, which correspond, respectively, to an "outer ring" and an "inner core" of the QD $[8,9]$. The two metallic regions are separated by 
(a)

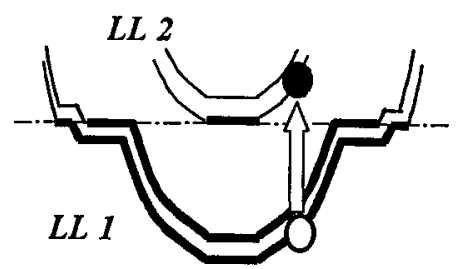

(b)

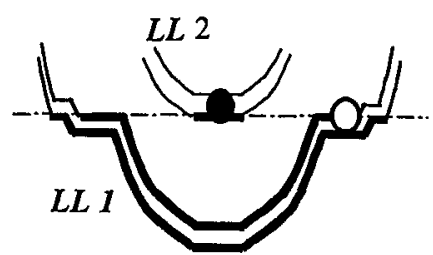

Fig. 2. Energy spectra of the lowest two orbital LLs in the QD. (a) Inter LL excitation due to absorption of a submillimeter photon. (b) The relaxed excited state with polarization.

an incompressible insulating strip. Accordingly, the tunnel probability of electrons between these two regions are strongly suppressed. It follows that the two metallic regions have different electrochemical potentials.

In our SET, the conductance resonance occurs when the electrochemical potential of the outer metallic ring lines up with that of the reservoirs. The QD functions as a strong absorber of submillimeter-waves if the photon energy is equal to the plasma-shifted cyclotron resonance (CR) energy. Upon the absorption of such a photon, as shown in Fig. 2a, the excited electron and the hole rapidly give up their excess energies to the lattice system and relax, respectively, into the inner core and the outer ring as shown in Fig. 2b. Thus the core and the ring are charged, respectively, by $-e$ and $+e$. This internal polarization leads to a reduction of the electrochemical potential of the outer ring by $\Delta \mu=\left(C_{2} / C_{12}\right) \varepsilon$ with the charging energy $\varepsilon=e^{2} /\left(C_{1}+C_{2}\right)$, where $C_{12}$ is the capacitance formed between the core and the ring, and $C_{2}\left(C_{1}\right)$ is the capacitance formed between the core (ring) and the surroundings of the QD. The reduction $-\Delta \mu \approx-\left(C_{2} / C_{12}\right) \varepsilon$ causes the conductance peak to shift towards the negative direction of the control gate voltage, $V_{\mathrm{g}}$. The ratio of the shift to the fundamental spacing between adjacent conductance resonance peaks (in the sweep of $V_{\mathrm{g}}$ ) is $C_{2} / C_{12} \approx 0.17$. Hence, the charge polarization induced by the single-photon absorption suffices to switch on (or off) the conductance resonance at a fixed $V_{\mathrm{g}}$. The switched-on (-off) state is maintained until the excited electron-hole pair recombines within the QD. In effect, this is equivalent to the amplification of a single photon to many electrons, the number of electrons being given by $\alpha=I_{\mathrm{DS}} \tau_{\mathrm{L}} / e$ with $I_{\mathrm{DS}}$ - the current transmitted through the SET (the $\mathrm{QD}$ ), $\tau_{\mathrm{L}}$ - the recombination lifetime of an electron-hole pair and $e-$ the unit charge. The lifetime $\tau_{\mathrm{L}}$ is very long, ranging from $10^{-3} \mathrm{~s}$ to $10^{3} \mathrm{~s}$ in the experiments, because the tunneling of an electron from the inner core region to the ring region is strongly suppressed as mentioned in the above. The "amplification coefficient $\alpha$ " in the experiments $\left(I_{\mathrm{DS}} \sim 100 \mathrm{pA}\right)$ reaches $10^{6}$ to $10^{12}$, which is comparable to or higher than that of photomultiplier tubes a vailable in the visible range.

\section{Experimental methods}

The metal gates along with their leads serve as a dipole antenna that couples submillimeter waves to the QD. The QD sample is installed in a ${ }^{3} \mathrm{He}-{ }^{4} \mathrm{He}$ mixing 


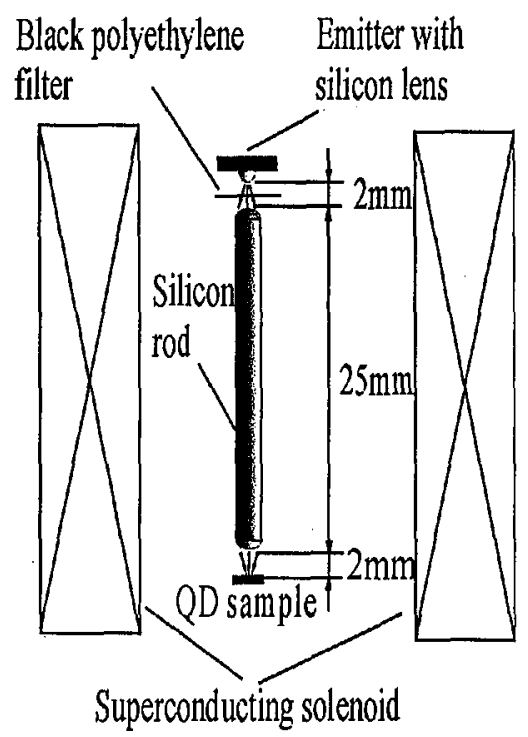

Fig. 3. Schematic view of the QD sample, the emitter, and the optical system.

chamber of a dilution refrigerator. As schematically shown in Fig. 3, a GaAs Hall bar is placed in the same mixing chamber to serve as a well-defined source of submillimeter waves [10]: when a current $I_{\text {emit }}$ is passed through the Hall bar, it emits a narrow-band cyclotron radiation at the frequency $\omega_{c}=\mathrm{eB} / \mathrm{m}^{*}$, where $m^{*}=0.068 m_{0}$ with the free electron mass $m_{0}$ is the effective electron mass. The emitted wave is guided through a $23 \mathrm{~mm}$ long silicon rod and illuminates the antenna for the QD. Unwanted radiation in the visible or near-infrared spectral range is completely eliminated by the silicon rod and black polyethylene filters. The submillimeter-wave power incident on the effective antenna area, $(0.1 \mathrm{~mm})^{2}$, is smaller than $\approx 0.003 \mathrm{fW}$ at $I_{\text {emit }}=3.5 \mu \mathrm{A}$ : in terms of the photon flux, it is less than $\approx 10^{4}$ photons $/ \mathrm{s}$.

\section{Experimental results}

Single submillimeter-wave photons were detected in a $B$ range of $3.4-4.15 \mathrm{~T}$, where the inner LL2 contains from twenty to one electrons. Figures $4 \mathrm{a}-\mathrm{c}$ exemplify the effect of illuminating the QD for one conductance peak at $T=0.07 \mathrm{~K}$ and $B=3.67 \mathrm{~T}$. The time constant of measurements is $1 \mathrm{~ms}$ and the gate voltage is scanned over three minutes for each curve. The conductance resonance located at $V_{\mathrm{g}}=-688.05 \mathrm{mV}$ in the absence of illumination (Fig. 4a) is occasionally switched off when very weak submillimeter waves are turned on $\left(I_{\text {emit }}=2 \mu \mathrm{A}\right)$ as shown in Fig. 4b. Complementarily, finite conductance occasionally shows up forming a few spikes distributed over a more negative $V_{\mathrm{g}}$ range. When the submillimeter wave is intensified $\left(I_{\text {emit }}=3.5 \mu \mathrm{A}\right.$ ), the original peak at $V_{\mathrm{g}}=-688.05 \mathrm{mV}$ almost vanishes leaving only a small number of narrow spikes, and is replaced by a dense array of conductance spikes that form a distinct envelope of another resonance 


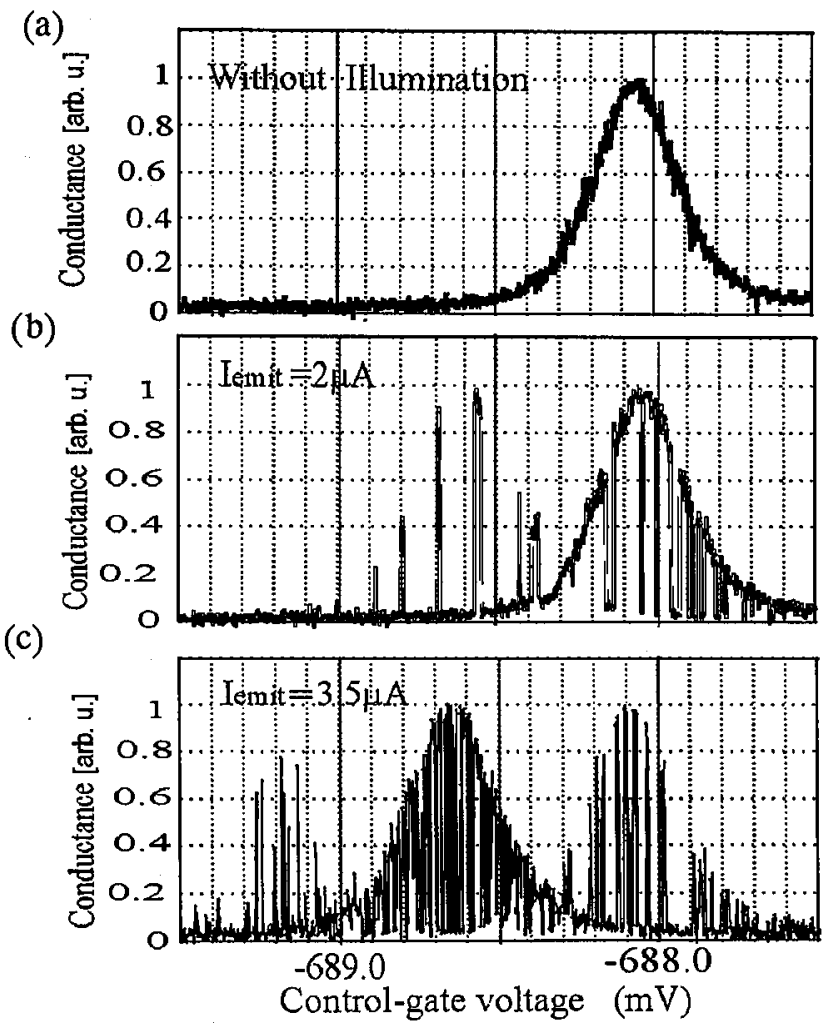

Fig. 4. Conductance through the QD as a function of $V_{\mathrm{g}}$ at $B=3.67 \mathrm{~T}$ and $T=70 \mathrm{mK}$. The scales of the conductance and $V_{\mathrm{g}}$ are the same for (a)-(c). (a) A regular conductance resonance in the absence of FIR illumination. (b) Under extremely weak illumination of submillimeter waves $(\lambda \approx 0.2 \mathrm{~mm})$. (c) Under weak illumination (but stronger than $(\mathrm{b})$ ).

line at $V_{\mathrm{g}}=-688.65 \mathrm{mV}$. The amplitude of the shift, $\Delta V_{\mathrm{g}}=-0.6 \mathrm{mV}$, is about $17 \%$ of the fundamental spacing of the conductance oscillations. The amplitude $\left(C_{2} / C_{12}=0.17\right)$ as well as the direction of the shift show that the switching of the conductance occurs due to the CR of the QD as expected.

When the gate voltage is fixed at the original peak position, the effect is seen as telegraph-like switches between two conductance states, as shown in Fig. 5 for $I_{\text {emit }}=3.5 \mu \mathrm{A}$. Each switching-off event corresponds to individual process of photon absorption by the QD, while each recovering process (switching-on event) corresponds to individual recombination process of an excited electron-hole pair within the QD. Although not shown here, the rate of switches increases with increasing the radiation intensity if the intensity is extremely weak $\left(I_{\text {emit }}<2.5 \mu \mathrm{A}\right)$. It levels off and starts decreasing at stronger illumination intensities $\left(I_{\text {emit }}>\right.$ $3.5 \mu \mathrm{A}$ ), where the average time interval between successive arrivals of incident photons (that are absorbed) becomes comparable to or shorter than the recombination lifetime of the electron-hole pair. 


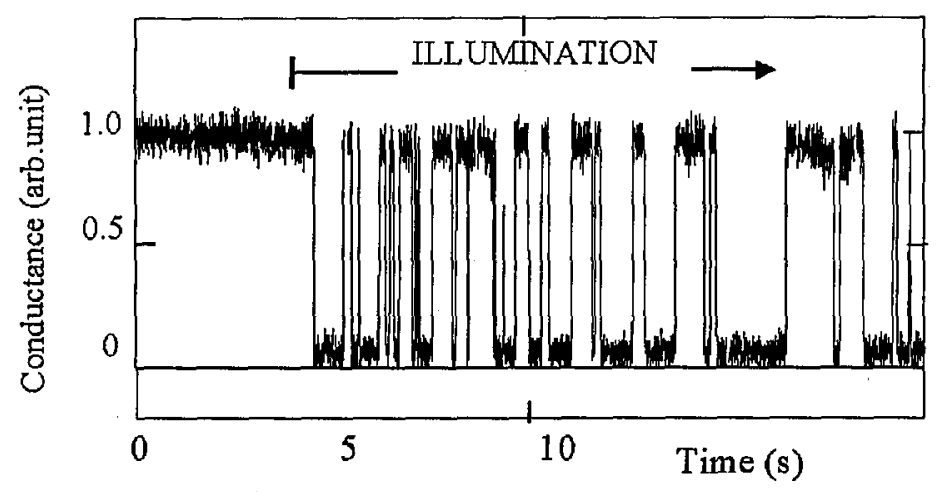

Fig. 5. Telegraph-like switches of conductance induced by a weak submillimeter illumination. $V_{\mathbf{g}}$ is fixed at the original peak position $(-688.05 \mathrm{mV})$. The other experimental conditions are the same as those of Fig. 4.

Compelling evidence that the conductance switches do indeed arise from CR of the QD $(B=3.60 \mathrm{~T})$ is shown in Fig. 6, where results of additional experiments are displayed [5]. Here submillimeter wave sources (a GaAs 2DEG Hall bar as well as an $n$-InSb device [11]) are installed in another superconducting solenoid placed outside the mixing chamber. This allows for independent tuning of the submillimeter wavelength. Figure 6 definitely shows that the emitter magnetic field, $B_{\mathrm{em}}$, which yields the largest count of switching corresponds to the "magnetoplasma resonance" frequency of the QD [12] that is slightly higher than the CR frequency of the bulk 2DEG (by about $3 \%$ ).

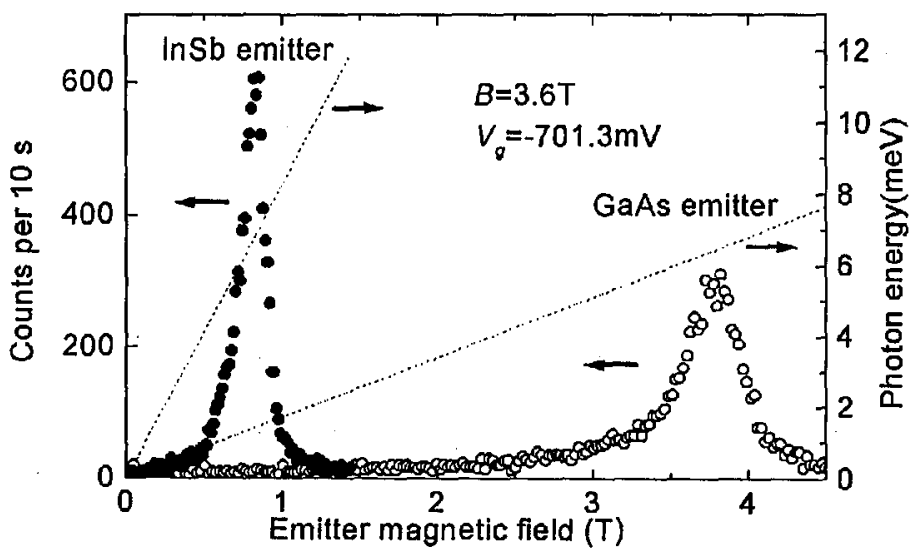

Fig. 6. Excitation spectra of the QD. The number of switching-off events of conductance over ten seconds, occurring when $V_{\mathrm{g}}$ is fixed at the original peak position, is shown against the magnetic field $B_{\text {em }}$ for the emitters. 


\section{Detector performance}

In the present work, single submillimeter wave photons were detected in the wavelength range of $0.17-0.2 \mathrm{~mm}$, which is restricted by the magnetic-field range (3.4-4.15 $\mathrm{T}$ ) where the recombination lifetime of excited electron-hole pairs exceeds the time constant of measurements $(1 \mathrm{~ms})$. Faster measurements will definitely expand the spectral range. The expansion of the spectrum may be done also by incorporating a back-gate structure into the $\mathrm{QD}$, which facilitates control of the optimal range of magnetic field.

We have confirmed that the conductance switches due to single photon absorption is sustained up to $T=0.4 \mathrm{~K}$, the upper bound being limited by the charging energy of the QD. Fabricating QDs on a narrow 2DEG mesa structure with minimum use of nearby metal gates may expand the upper bound to $T \approx 0.8 \mathrm{~K}$, making possible the use of more convenient ${ }^{3} \mathrm{He}$ refrigerators.

Very few conductance switches were seen if the device was not illuminated. The "dark signal" in terms of the switch rate was smaller than $0.001 / \mathrm{s}$ at $T=0.07 \mathrm{~K}$ in an optimal $B$ range.

The quantum yield of the present $\mathrm{QD}$ detector, or the ratio of the photon count to the incident photon flux falling on the effective antenna area, is roughly estimated to be one per cent. The quantum yield, which crucially depends on the architecture of optics, may be improved significantly in the future.

The effective NEP in the present non-optimized scheme is roughly on the order of $10^{-22} \mathrm{~W} / \mathrm{Hz}^{1 / 2}$, which is superior to any other conventional detectors reported in the literature [1-4] by a factor more than $10^{4}$.

\section{Discussion and conclusion}

The outstanding sensitivity of our QD detector may be understood in the following way. The inner core of the QD can be regarded as a built-in floating gate for the SET. One extra electron is added to this "inner-core gate" upon CR. Single extra electron on the "inner-core gate" suffices to switch off (or on) the SET, and it survives in the "inner-core gate" for such a long time as to allow for direct detection of this switching event. In fact, the recombination lifetime of an electron excited in the inner core exceeds $1 \mathrm{~ms}$ and reaches as long as $1300 \mathrm{~s}$ in an optimal $B$ range [6]. It follows that one absorbed photon in our QD detector leads to a current of $10^{6}-10^{12}$ electrons. This is in marked contrast to other mechanisms of conventional detectors [1-4] or to the well-known photon-assisted tunneling in SETs [5], in which one photon only transfers, at most, a few electrons.

\section{Acknowledgments}

This work was supported by the Core Research for Evolutional Science and Technology (CREST) of the Japan Science and Technology Corporation (JST). We appreciate helpful and enjoyable discussion with M. Stopa. 


\section{References}

[1] M. Fujiwara, M. Hiromoto, Jpn. J. Appl. Phys. 36, 4262 (1997).

[2] J. Kawamura, R. Blundell, C.Y.E. Tong, G. Goltsman, E. Gershenzon, B. Voronov, S. Cherednichenko, Appl. Phys. Lett. 70, 1619 (1997).

[3] J.R. Tucker, M.J. Feldman, Rev. Mod. Phys. 57, 1055 (1985).

[4] R.J. Schoelkopf, S.H. Moseley, C.M. Stahle, P. Wahlgren, P. Delsing, IEEE Trans. Appl. Supercond. 9, 2935 (1999).

[5] S. Komiyama, O. Astafiev, V. Antonov, T. Kutsuwa, H. Hirai, Nature 403, 405 (2000).

[6] V. Antonov, O. Astafiev, T. Kutsuwa, H. Hirai, S. Komiyama, Physica E 6, 367 (2000).

[7] S. Komiyama, O. Astafiev, V. Antonov, T. Kutsuwa, Physica E 7, 698 (2000).

[8] L.P. Kouwenhoven, C.M. Marcus, P.L. McEuen, S. Tarucha, R.M. Westervelt, N.S. Wingreen, in: Mesoscopic Electron Transport, Eds. L.L. Sohn, L.P. Kouwenhoven, G. Schön, Kluwer Academic Publ., Dordrecht 1997, p. 105.

[9] N.C. van der Vaart, L.P. Kouwenhoven, M.P. de Ruyter van Steveninck, Y.V. Nazarov, C.J.P.M. Harmans, C.T. Foxon, Phys. Rev. B 55, 9746 (1997).

[10] Y. Kawano, Y. Hisanaga, S. Komiyama, Phys. Rev. B 59, 12537 (1999).

[11] E. Gornik, W. Muller, H.W. Potzl, G. Bauer, M. Overhamm, P. Grosse, Infrared Phys. 16, 285 (1976).

[12] K. Bollweg, T. Kurth, D. Heitmann, V. Gudmundsson, E. Vasiliadou, P. Grambow, K. Eberl, Phys. Rev. Lett. 76, 2774 (1996). 